\title{
Efecto de diferentes láminas de riego en el crecimiento y desarrollo de cebolla de bulbo (Allium cepa L.)
}

\section{Effect of different water levels on the growth and development of bulb onion (Allium cepa L.)}
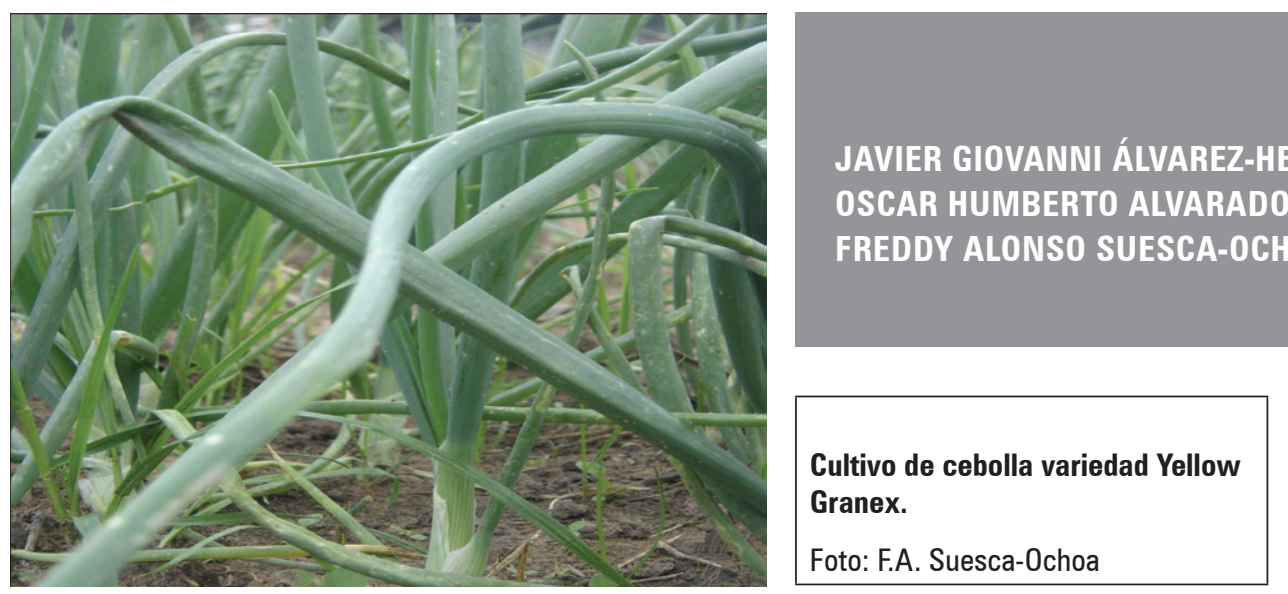

\section{RESUMEN}

Debido al sistema radicular superficial, la cebolla de bulbo requiere de un suministro de riego en el momento y con la intensidad adecuada para favorecer la absorción de agua y nutrientes y obtener altos rendimientos. Se evaluó el efecto de diferentes láminas de riego en el crecimiento y desarrollo de cebolla de bulbo en un suelo franco arenoso y con un sistema de riego por aspersión. Se usó un diseño experimental completamente al azar con cuatro tratamientos (coeficientes de cultivo de 0,8; 1,0; 1,2 y 1,4 de la evaporación (Ev)), cada uno con 16 repeticiones, para un total de 64 unidades experimentales, donde cada unidad experimental correspondió a una parcela de $1 \mathrm{~m}^{2}$. El coeficiente de 1,2 (43 L por planta por ciclo) presentó los mayores promedios en masa seca total y masa seca del bulbo con diferencias significativas en relación al coeficiente de 0,8 , pero sin diferencias con la aplicación de 1,0 y 1,4 de la Ev. Además, en masa seca de raíz el tratamiento de 1,2 Ev presentó el mayor promedio. En el crecimiento general no hubo diferencias significativas los primeros 70 días después del trasplante, pero luego de este tiempo se presentaron diferencias estadísticas y la aplicación de 1,2 de la Ev presentó la mayor acumulación de masa seca.

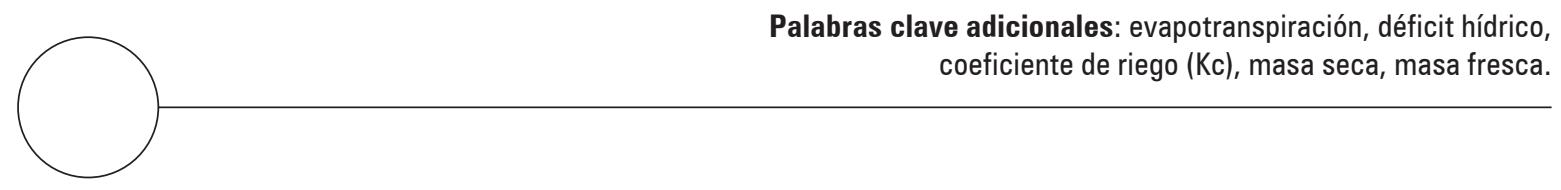

\footnotetext{
Facultad de Ciencias Agropecuarias, Grupo de Investigaciones Agrícolas, Universidad Pedagógica y Tecnológica de Colombia (UPTC), Tunja (Colombia). ORCID Álvarez-Herrera, J.: 0000-0002-1737-6325; ORCID Alvarado-Sanabria, O.H.: 0000-0002-5833-8405; ORCID Suesca-Ochoa, F.A.: 0000-0003-0238-2770

2 Autor para correspondencia. javier.alvarez@uptc.edu.co
} 


\section{ABSTRACT}

Because of the shallow root system of the onion plant, it requires irrigation at the proper time and intensity in order to promote the absorption of water and nutrients to obtain a high yield. We evaluated the irrigation rate effect on onion growth and development in a sandy loam soil with a sprinkler irrigation system. The experiment design was completely randomized with four treatments (evaporation coefficients of 0.8, 1.0,1.2 and $1.4 \mathrm{of}(\mathrm{Ev})$ ), each with 16 repetitions, for 64 experiment units; each experiment unit was a $1 \mathrm{~m}^{2}$ plot. The 1.2 coefficient ( $43 \mathrm{~L}$ per plant per cycle) showed the highest total dry mass and dry mass of the bulb with significant differences from the 0.8 coefficient, but no differences with the application of the 1 and $1.4 \mathrm{Ev}$. Furthermore, the $1.2 \mathrm{Ev}$ treatment showed the highest root dry mass. There was no significant difference between the treatments during the first 70 days after transplant, but after this time, there was a statistically difference and the 1.2 Ev showed the highest dry matter accumulation.

Additional key words: evapotranspiration, water deficit, irrigation coefficient (Kc), dry mass; fresh mass.

Fecha de recepción: 20-03-2017 Aprobado para publicación: 30-09-2017
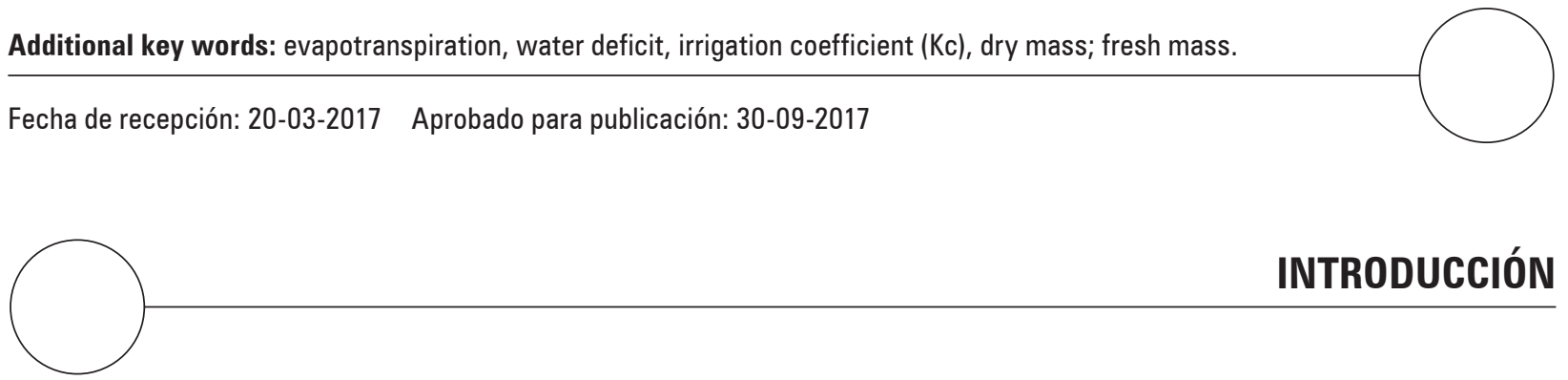

La cebolla de bulbo ocupa el cuarto lugar en producción mundial de hortalizas, ya que alcanzó una producción de 84 millones de toneladas en el 2013 (FAO, 2016), lo que resalta la importancia de este cultivo a nivel mundial. En Colombia, los principales departamentos productores de cebolla de bulbo son: Boyacá con 130.000 t, que corresponde al 51\% de la producción nacional, Norte de Santander con 31.200 t $(22,6 \%)$ y Cundinamarca con 29.438 t $(19,6 \%)$ (AGRONET, 2016). Entre Cundinamarca y Boyacá representan el 68,6\% del área sembrada en el país con 8.000 ha aproximadamente, lo que muestra la importancia de este sistema productivo en el altiplano cundiboyacense.

Colombia presenta un bajo rendimiento en la producción de cebolla de bulbo con 23,3 t ha ${ }^{-1}$, el cual es inferior al de otros países suramericanos como Chile $\left(44 t \mathrm{tha}^{-1}\right)$, Perú $\left(39 \mathrm{t} \mathrm{ha}^{-1}\right)$, Argentina $\left(29 \mathrm{t} \mathrm{ha}^{-1}\right)$, Venezuela $\left(24,8 \mathrm{t} \mathrm{ha}^{-1}\right)$ y Brasil (26 t ha-1 $)$ (FAO, 2016); lo que demuestra un atraso en la generación y aplicación de tecnologías de producción. Una gran limitante es la disponibilidad de riego, ya que en otros países un adecuado suministro de agua aumenta la producción hasta en un 200\% (Bekele y Tilahun, 2007), por lo que un apropiado manejo del recurso hídrico, mediante la implementación de sistemas de riego eficientes, garantizaría un mayor rendimiento en el cultivo de la cebolla en nuestro país.
La finalidad del riego es suministrar a los cultivos el agua -adicional a la precipitación- requerida para el crecimiento óptimo de manera uniforme. Esta cantidad de agua depende de condiciones climáticas, las cuales están representadas en la evapotranspiración de un cultivo de referencia (ETo), y más específicamente en la evapotranspiración del cultivo de cebolla bajo condiciones estándar (ETc), ésta se refiere al flujo de evaporación que demanda la atmósfera sobre los cultivos que son sembrados en grandes áreas sin sombrío, bajo niveles óptimos de agua en el suelo y un correcto manejo agronómico (FAO, 2006). Teniendo en cuenta estos dos factores se establece la cantidad y frecuencia del riego para obtener un buen desarrollo y rendimiento del cultivo de cebolla.

Estudios sobre riego en este cultivo se hacen principalmente en zonas áridas y templadas, y se enfocan en el efecto sobre el rendimiento y calidad poscosecha. Con base en un estudio realizado en Texas, Enciso et al. (2009) demostraron que la aplicación de láminas de riego subsuperficial del 70 y $100 \%$ de la Ev no afectan los rendimientos significativamente, mientras que, con el $50 \%$ de la Ev, estos rendimientos disminuyen en un $20 \%$. En otro estudio realizado por Bekele y Tilahun (2007) en Etiopia, se determinó que, ante un déficit hídrico, es mejor disminuir la cantidad de agua aplicada durante todo el ciclo del cultivo que eliminar totalmente el riego durante alguna etapa fenológica. 
Los estudios sobre láminas de riego en el cultivo de cebolla de bulbo, bajo condiciones del trópico alto son escasos; por lo tanto, el presente trabajo pretende determinar el efecto de diferentes láminas de riego sobre el crecimiento, desarrollo y producción de cebolla de bulbo.

\section{MATERIALES Y MÉTODOS}

El experimento se realizó a campo abierto en la granja Las Flores de la Universidad Pedagógica y Tecnológica de Colombia localizada en la ciudad de Tunja (Boyacá), la cual se encuentra a una altitud de 2.782 msnm, $5^{\circ} 32^{\prime} \mathrm{N}$ y $73^{\circ} 23^{\prime} \mathrm{W}$, temperatura promedio de $13^{\circ} \mathrm{C}$, humedad relativa del $70 \%$ y una precipitación promedio anual de $760 \mathrm{~mm}$. El suelo de la zona de estudio presenta una textura franco-arenosa, con un contenido de arena del 65,42\%, limo de $18 \%$ y arcilla del $16,58 \%$, un drenaje externo rápido e interno moderado.

Se usaron plántulas de la especie Allium cepa L. variedad Yellow Granex, a las cuales se les cortó un tercio de la parte aérea y de la raíz y fueron sembradas a una distancia de $10 \mathrm{~cm}$ entre plantas y entre surcos. Se usó un diseño experimental completamente al azar, donde se evaluó la aplicación de cuatro láminas de riego (coeficientes multiplicadores de la evapotranspiración $0,8,1,0,1,2$ y 1,4) con 16 repeticiones para un total de 64 unidades experimentales (UE); cada UE corresponde a una parcela de $1 \mathrm{~m}^{2}$ con 36 plantas. Durante el ensayo se realizó una fertilización con $90 \mathrm{~kg}$ ha- ${ }^{-1}$ de $\mathrm{N}, 90 \mathrm{~kg} \mathrm{ha}^{-1}$ de $\mathrm{P}_{2} \mathrm{O}_{5}$ y $90 \mathrm{~kg} \mathrm{ha}^{-1}$ de $\mathrm{K}_{2} \mathrm{O}$ con el fin de evitar deficiencias nutricionales.

A los 12 días después del trasplante (ddt) y mediante un sistema de riego por aspersión, se inicio la aplicación de los tratamientos con una frecuencia de $4 \mathrm{~d}$, teniendo en cuenta la ecuación 1.

$$
\boldsymbol{\mu}_{\mathrm{c}}=\frac{(\mathrm{Ev}-\mathrm{P})^{*} \mathrm{C}^{*} \mathrm{~A}}{\eta_{\mathrm{r}}}
$$

donde, $\mu_{c}$ es la cantidad de agua a aplicar (mm), Ev la evaporación en mm medida en el tanque evaporímetro clase A, $P$ precipitación acumulada $(\mathrm{mm}), C$ coeficiente multiplicador de la evapotranspiración, $A$ área de las parcelas, $\eta_{\mathrm{r}}$ eficiencia del riego del riego por aspersión $(0,7)$.

Para establecer las curvas de crecimiento de cada tratamiento, se tomaron 10 plantas al azar de cada UE a los 7, 14, 43, 77, 105 y 129 ddt. Estas plantas se secaron a $70^{\circ} \mathrm{C}$ durante $72 \mathrm{~h}$ para luego determinarles la masa seca con una balanza electrónica Acculab VIC 612 (Sartorius Spain, Madrid, España). Después de los 130 d se determinó la masa seca total, masa seca de raíces de las plantas y se cosecharon los bulbos, a los cuales se les midió la masa fresca y masa seca. Luego para cada tratamiento, se estableció el crecimiento de la masa seca mediante un modelo logístico sigmoideo (Ec. 2).

$$
y=\frac{a}{1+e^{(-b t-c)}}
$$

donde, $y$ es la masa seca de la planta en g, a es la máxima masa seca acumulada durante el tiempo $(t)$ de duración de la fase de cultivo, $b$ y c son constante relacionadas con la velocidad relativa de crecimiento y el punto de inflexión del modelo.

Se hizo un análisis de varianza (Anova) para cada variable, con el fin de determinar la existencia de diferencias estadísticas entre tratamientos, y se realizó la prueba de comparación de medias de Tukey al 5\%, para lo cual se uso el software SAS v. 8.1e (Cary, N.C).

\section{RESULTADOS Y DISCUSIÓN}

\section{Masa fresca total (MFT) y masa seca total (MST)}

Para la MST, a los 129 ddt se presentaron diferencias significativas $(P \leq 0,05)$ entre los coeficientes de riego de 1,2 y 0,8 pero no entre los demás tratamientos. E1 mayor promedio lo presentó la aplicación de 1,2 de la Ev (Fig. 1). En la variable MFT se presentó el mismo comportamiento, pero sin diferencias significativas entre todos los tratamientos. Esta mayor acumulación de masa seca con el coeficiente de riego de 1,2 de la Ev concuerda con lo reportado por Kumar et al. (2007a) quienes al usar riego por microaspersión en un suelo arenoso encontraron que con riegos del 100 y $120 \%$ de la Ev hay una mayor acumulación de masa seca que con 60 y $80 \%$.

La acumulación progresiva de masa seca conforme aumenta el coeficiente de riego desde 0,8 hasta 1,2 se debe a que las plantas tienen una mayor cantidad de agua aprovechable en el suelo. El agua es parte fundamental del crecimiento y desarrollo de la planta y constituye el medio de transporte de moléculas indispensables para la vida como proteínas, ácidos nucleicos, polisacáridos y otros componentes de la célula 


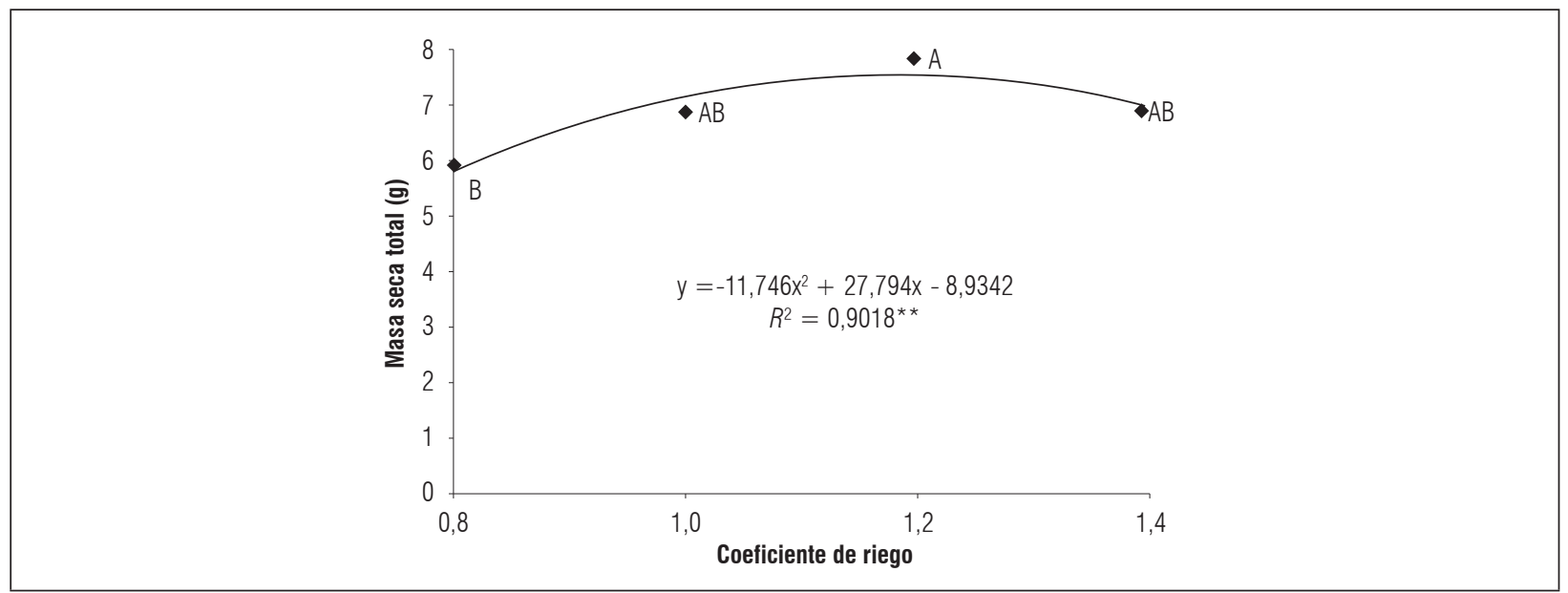

Figura 1. Efecto del coeficiente de riego en la materia seca total de plantas de cebolla 'Yellow Granex' a los 129 d después del trasplante en Tunja, Colombia. Promedios con letras distintas indican diferencia significativa según la prueba de Tukey $(P \leq 0,05), n=16$.

(Taiz et al., 2015). Por otro lado, las plantas pierden continuamente agua por medio de la transpiración, lo cual les permite regular temperatura e intercambiar vapor de agua por $\mathrm{CO}_{2}$, fundamental para que se lleve a cabo la fotosíntesis (Medrano et al., 2008). Estos procesos garantizan un mayor crecimiento (Rattin et al., 2011), representado en una mayor acumulación de masa seca cuando se aplica mayor cantidad de agua.

El menor crecimiento originado por la aplicación del coeficiente de 1,4 en relación al de 1,2 podría deberse a la saturación de humedad en el suelo provocada por una cantidad de agua superior a la que se puede infiltrar, esta saturación disminuye el contenido de oxígeno y provoca anoxia en la planta (Sauter, 2013). Bajo condiciones de anoxia la planta produce una mayor cantidad de ácido aminociclopropano carboxílico (ACC), el cual es transportado desde las raíces hacia la parte aérea de la planta donde se convierte en etileno (Irfan et al., 2010). Dentro de los efectos del etileno esta la ralentización del crecimiento y la abscisión foliar, provocando una menor acumulación de masa seca bajo un riego excesivo (Steffens, 2014), lo que explicaría el menor crecimiento bajo el coeficiente 1,4.

Kumar et al. (2007b) reportan que en un suelo arenoso y usando un sistema de microaspersión la aplicación del $100 \%$ de la Ev tiene el mismo efecto que aplicar un $120 \%$ sobre la acumulación de masa seca total de la planta. Por otro lado, Metwally (2011) estudió un suelo arcilloso de Australia y reporta que el número de hojas y la masa seca de las mismas es menor bajo un riego de $1,75 \mathrm{~m}^{3}$ por metro cuadrado que uno de
$0,9 \mathrm{~m}^{3}$. Esto muestra que la cantidad de agua aplicada tiene un efecto favorable en el crecimiento; sin embargo, este tiene un límite, luego del cual se afecta negativamente el crecimiento del cultivo.

\section{Masa seca de raíces (MSR)}

Después de 129 d, hubo diferencias significativas para la MSR entre el coeficiente de 1,2 y los demás coeficientes (Fig. 2) con un 37\% más de masa seca que el promedio de los demás tratamientos. Esta variable tiene la misma tendencia que la acumulación de masa seca total, lo que muestra la estrecha relación entre el desarrollo radicular y el desarrollo general de la planta.

Un escaso suministro de agua provoca un estrés hídrico y por tanto una menor acumulación de masa seca radicular en los tratamientos con la aplicación de riego con un coeficiente multiplicador de la evaporación de 0,8 y 1,0 (Fig. 2), lo que indica que un suministro con el $100 \%$ de la evapotranspiración durante todo el ciclo del cultivo no garantiza un desarrollo radicular óptimo. Lo anterior concuerda con lo reportado por Shao et al. (2008) en varias especies, donde un estrés hídrico provoca un desarrollo radicular lento, en el cual se presentan raíces cortas y gruesas, las cuales no absorben gran cantidad de agua debido a su baja área superficial.

Así mismo, la menor acumulación de MSR con el coeficiente de 1,4 se debe a que cuando el suelo esta saturado, los gases como el $\mathrm{O}_{2}$ y el $\mathrm{CO}_{2}$ se difunden 


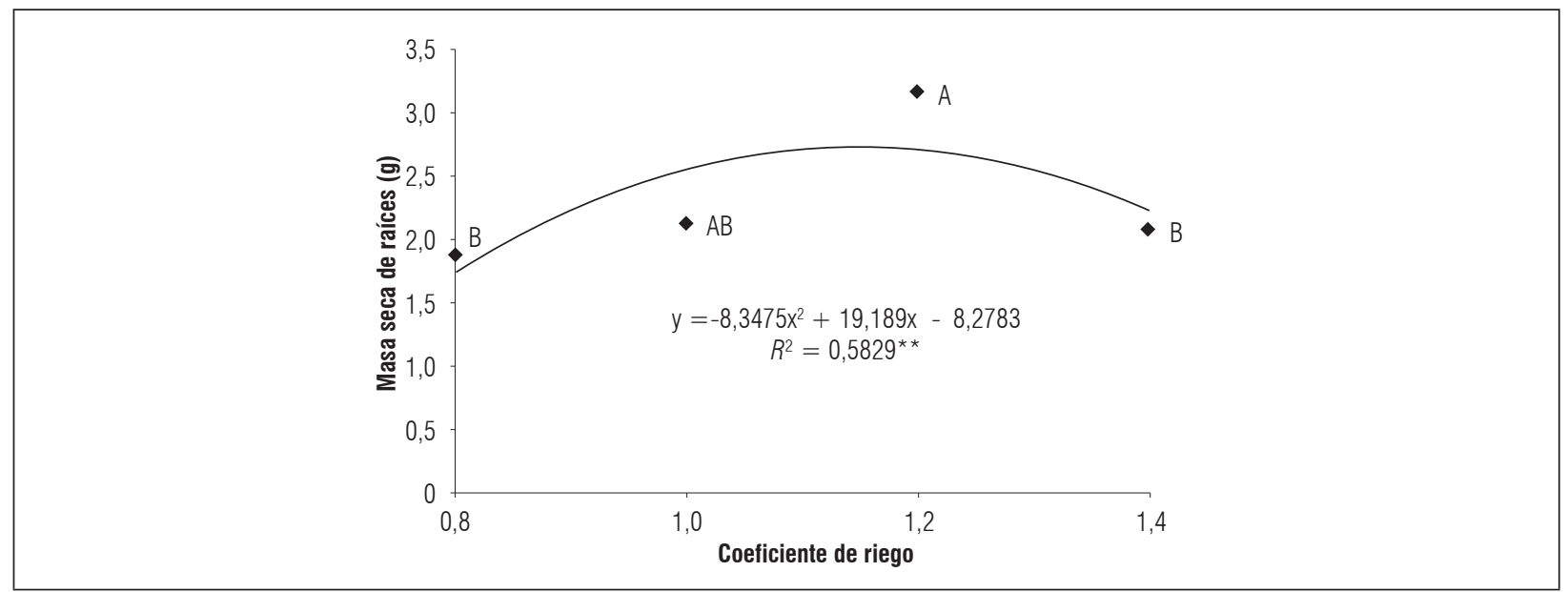

Figura 2. Efecto del coeficiente de riego sobre la masa seca de la raíz de cebolla 'Yellow Granex' a los 129 d después del trasplante en Tunja, Colombia. Promedios con letras distintas indican diferencia significativa según la prueba de Tukey $(P \leq 0,05), n=16$.

más lentamente en el agua haciéndose inaccesibles para las raíces de la planta (Taiz et al., 2015). Con la falta de oxígeno disminuye la producción de moléculas energéticas como el ATP, lo que provoca una reducción del metabolismo radicular aminorando la absorción de agua y nutrientes (Sauter, 2013). Esta falta de nutrientes y agua explicaría la menor acumulación de masa seca cuando el agua aplicada es mayor a 1,2 de la Ev.

\section{Curvas de crecimiento}

En los primeros $77 \mathrm{ddt}$ no hay diferencias estadísticas significativas en la acumulación de masa seca entre las distintas láminas de riego aplicadas (Fig. 3). Esto significa que en el primer estadio del cultivo las plantas no necesitan mayor cantidad de agua y suplen sus necesidades con la aplicación de 0,8 de la Ev, lo que coincide con Bekele y Tilahun (2007) quienes determinaron que un déficit hídrico en la etapa inicial provocado al aplicar el $25 \%$ menos de la Ev no afecta el rendimiento de forma significativa, en comparación con un riego del $100 \%$ de la Ev. Por lo que se podría afirmar que en las primeras 10 semanas y bajo las condiciones evaluadas, el cultivo de cebolla no demanda más del 80\% de la evapotranspiración.

A los 80 ddt se observaron diferencias significativas entre el coeficiente de 1,2 (con una masa seca de 6,48 g) y los tratamientos de 0,8 y 1,0 de la Ev (masa seca de 5,03 y 5,16 g, respectivamente), pero sin diferencias con 1,4 de la Ev (Fig. 3), lo que indica que a partir de esta fecha el cultivo demanda una mayor cantidad de agua para el normal desarrollo y concuerda con Kumar et al. (2007a), quienes después de 75 d observaron diferencias significativas en la masa seca entre láminas de 0,6 y 0,8 de la Ev, en relación con las de 1,0 y $1,2 \mathrm{Ev}$, las cuales presentaron una mayor masa. Se puede inferir que luego de los 80 ddt la aplicación de 1,2 Ev es suficiente para un óptimo desarrollo del cultivo de cebolla.

Un aspecto relevante durante la etapa de crecimiento del cultivo en la variable masa seca a los $105 \mathrm{ddt}$ fue la pérdida de una parte del área foliar por la presencia de los patógenos Peronospora destructor y Alternaría porri. Hay que tener en cuenta que el agua sobre las hojas pudo favorecer el desarrollo de enfermedades causadas por hongos (Mishra et al., 2014), lo cual sumado a la alta densidad de siembra (1.10 $\mathrm{plantas} / \mathrm{ha})$ y la humedad relativa (mayor al 75\%) presente en esta época de desarrollo del cultivo, aumentó la susceptibilidad del cultivo a estos patógenos, lo que explica que la masa seca se mantuviera constante entre los días 77 y 105 en todos los tratamientos.

Aunque durante los últimos $25 \mathrm{~d}$ solo hubo diferencias estadísticas significativas entre dos coeficientes 1,2 y 0,8 , es interesante que en esta última etapa los dos coeficientes extremos $(0,8$ y 1,4$)$ presentaron aproximadamente la misma masa seca que a los 105 días, mientras que los tratamientos de 1,0 y $1,2 \mathrm{Ev}$ mostraron un aumento. Esto podría significar que en la última etapa un exceso o déficit de agua tiene un efecto negativo sobre la maduración del bulbo, el cual 


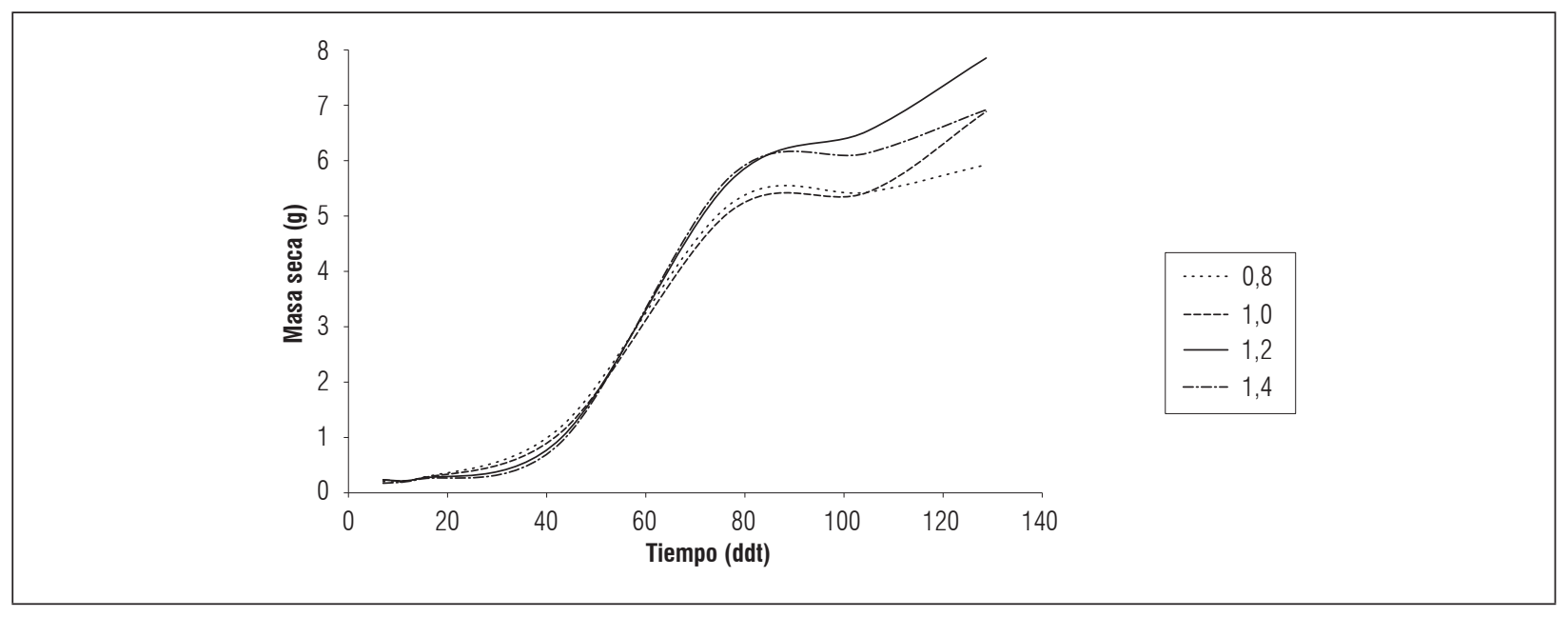

Figura 3. Curvas de crecimiento o tasa relativa de crecimiento acumulado de plantas de cebolla 'Yellow Granex' bajo distintos coeficientes de riego en Tunja, Colombia.

es la expresión del último estadio fenológico del cultivo (Brewster, 2008).

Considerando que las necesidades hídricas del cultivo dependen de la transpiración de la planta y de la evaporación de agua del suelo, se infiere que en los primeros 70 días, la baja evapotranspiración se debe a que la evaporación en el suelo y la transpiración de la planta (con una baja área foliar en esta etapa) no superan el $80 \%$ del agua evapotranspirada por un cultivo de referencia (ETo). Sin embargo, luego de 80 días cuando la planta tiene la mayor parte de sus hojas desarrolladas, el cultivo requiere mayor cantidad de agua ya que aumenta la transpiración y comienza la formación del bulbo (Brewster, 2008). Estos requerimientos provocan que la planta no alcance su máximo crecimiento cuando el suministro de agua es inferior a 1,2 de la Ev. A los 90 ddt, la aplicación de 1,2 de la Ev es suficiente para un buen desarrollo, mientras que una cantidad mayor afecta negativamente la acumulación de masa seca, lo que concuerda con la FAO (2006), la cual afirma que durante la etapa final del cultivo las necesidades hídricas disminuyen en relación con la época en la que el cultivo se encuentra en estado vegetativo, por lo que demanda mayor cantidad de agua en las etapas de desarrollo y final de cosecha en comparación con la etapa inicial.

\section{Modelo de crecimiento logístico sigmoideo para cebolla de bulbo}

El modelo logístico sigmoideo para cada tratamiento muestra los parámetros hallados mediante una regresión no lineal (Tab. 1). El parámetro $a$, que corresponde a la máxima masa seca alcanzada, mostró diferencias significativas entre el coeficiente de 1,2 con el mayor valor y el de 0,8 con el menor. Por otro lado, el parámetro $c$ (día en que la velocidad de crecimiento fue mayor y a partir del cual el crecimiento comenzó a disminuir) presentó diferencias estadísticas significativas, ya que el coeficiente de 0,8 fue el tratamiento que más rápido presentó el punto de inflexión (58ddt), mientras que la aplicación de un coeficiente de riego de 1,2 mostró el punto de máximo crecimiento después (69 ddt). Esto permite determinar que las plantas de cebolla del tratamiento con la aplicación de un coeficiente de 0,8 alcanzan su máxima velocidad de crecimiento más rápido, no obstante, la velocidad de crecimiento disminuye de manera más rápida comparada con los demás tratamientos; del mismo modo, se acumula menor masa seca, lo que se ratifica con un valor bajo de $c$, el cual es mayor en

Tabla 1. Parámetros del modelo logístico sigmoideo para cebolla 'Yellow Granex' bajo distintos coeficientes de riego en Tunja, Colombia.

\begin{tabular}{|c|c|c|c|}
\hline $\begin{array}{c}\text { Coeficiente } \\
\text { multiplicador de la Ev }\end{array}$ & a (g) & b $\left(\mathrm{g} \mathrm{dia}^{-1}\right)$ & $\mathrm{C}(\mathrm{ddt})$ \\
\hline 0,8 & $6,13 \mathrm{~b}$ & $0,086 \mathrm{a}$ & $58,17 \mathrm{~b}$ \\
\hline 1,0 & $7,1 \mathrm{ab}$ & $0,090 \mathrm{a}$ & $63,94 \mathrm{ab}$ \\
\hline 1,2 & $7,98 \mathrm{a}$ & $0,078 \mathrm{a}$ & $69,43 \mathrm{a}$ \\
\hline 1,4 & $6,71 \mathrm{~b}$ & $0,084 \mathrm{a}$ & $63,41 \mathrm{ab}$ \\
\hline
\end{tabular}

ddt: días después del trasplante. Promedios con letras distintas indican diferencia significativa según la prueba de Tukey $(P \leq 0,05), n=16$. 

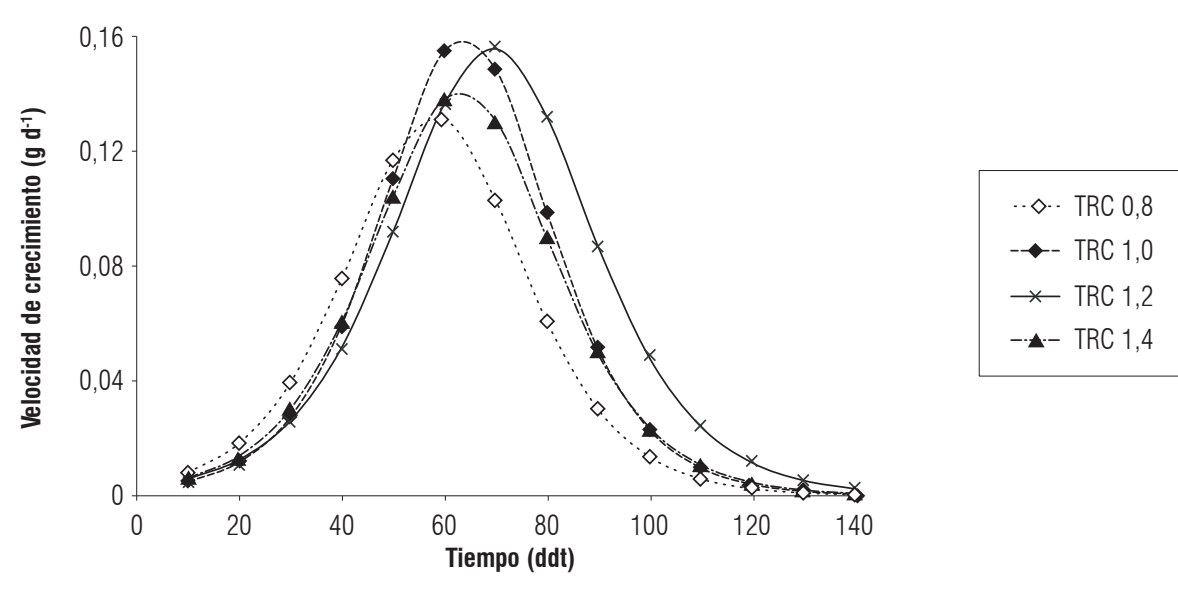

Figura 4. Curvas de velocidad de crecimiento o tasa relativa de crecimiento (TRC) de cebolla 'Yellow Granex' bajo distintos coeficientes de riego en Tunja, Colombia.

el coeficiente de 1,2 que con el de 0,8, y presentó la menor acumulación de masa seca.

Aunque en el parámetro $b$ no se presentaron diferencias significativas que se relaciona con la velocidad de crecimiento (Fig. 4). Como lo reporta Zheng et al. (2012), la primera etapa se caracteriza por una mayor tasa de crecimiento, luego se alcanza un pico (parámetro c) en la velocidad de crecimiento para finalmente continuar con la senescencia de la planta. Se puede apreciar también que la máxima tasa de crecimiento en los primeros $50 \mathrm{~d}$ la obtuvo el coeficiente de 0,8 , entre los 50 a los $72 \mathrm{ddt}$, fue el coeficiente de 1,0 el de mayor crecimiento. Después de los $72 \mathrm{~d}$, regar con una lámina de riego de 1,2 de la evaporación mostró los mejores resultados, lo cual indica que estos son los coeficientes de riego más adecuados para las distintas etapas.

El comportamiento sigmoideo consta de tres etapas; fase logarítmica, lineal y de senescencia y cada una de estas etapas es afectada genética y ambientalmente (Thomas, 2013). En este caso las distintas láminas de riego afectaron el inicio de la etapa de senescencia, la cual comenzó inicialmente en los tratamientos con coeficiente de 0,8 y 1,0 que cuando se aplicó 1,2 y 1,4 de la Ev. Esta senescencia temprana ha sido reportada por otros autores como Kumar (2007a), quien encontró que la etapa de senescencia se daba primero en los tratamientos de 0,6 y 0,8 de la Ev y luego en los tratamientos de 1,0 y 1,2 de la Ev, estos últimos, también acumularon la mayor masa seca. Es por esto que, cuando la planta se somete a un estrés hídrico esta presenta una cadena de reacciones en las células vegetales que termina en la producción de hormonas como: el ácido abscísico (ABA), ácido salicílico y etileno. Este último provoca la senescencia de la planta (Voesenek y Sasidharan, 2013); lo que explica que este proceso comience en los tratamientos con la menor cantidad de agua aplicada (coeficiente de 0,8 y 1,0 ).

\section{Masa fresca de bulbo (MFB) y masa seca de bulbo (MSB)}

La MSB presentó diferencias estadísticas entre los coeficientes de 0,8 y 1,2. La aplicación del coeficiente de riego de 1,2 en cebolla de bulbo generó la mayor acumulación de masa seca. En cuanto a la MFB, la lámina de riego de 1,4 de la Ev mostró la mayor acumulación de masa fresca, pero sin diferencias significativas entre tratamientos. La relación entre coeficientes y masa fresca es lineal (Fig. 5), mientras que en la MSB hay una tendencia logarítmica, esto sugiere que al aumentar la cantidad de agua hasta el 140\% de la Ev, se garantiza una mayor masa fresca del bulbo, pero la acumulación de solutos se mantiene constante desde el coeficiente 1,2.

Estos resultados también son reportados por Kumar et al. (2007a) en Etiopía, quienes después de 105 días bajo coeficientes de riego de 0,6 y 0,8 de la Ev observaron una masa fresca de bulbo más baja, con 29 y $41 \mathrm{~g}$, respectivamente por bulbo, mientras que con coeficientes de 1,0 y 1,2 se obtuvo una masa fresca de bulbo de 48 y 52 g, respectivamente. Esto muestra que la masa fresca del bulbo aumenta con la cantidad de agua aplicada, por lo menos hasta una aplicación de 1,4 de la Ev. 


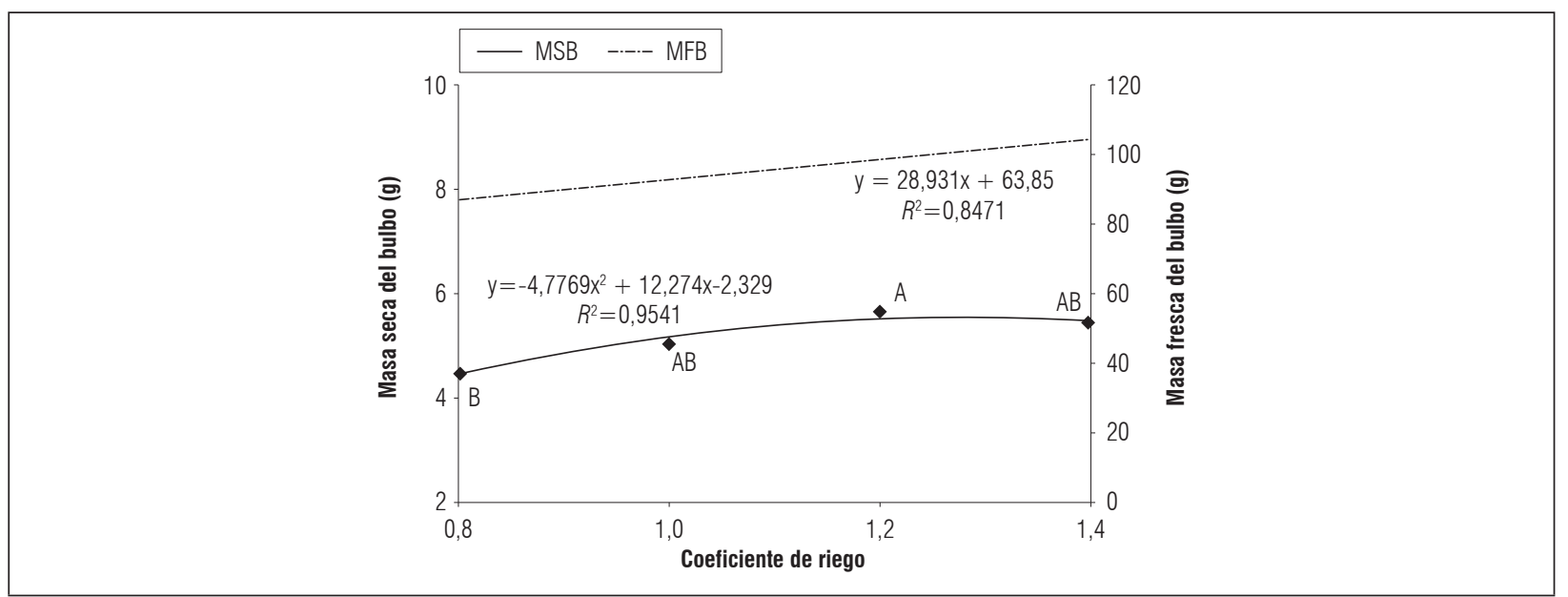

Figura 5. Efecto del coeficiente de riego en la masa fresa y seca del bulbo de cebolla 'Yellow Granex' bajo distintos coeficientes de riego en Tunja, Colombia. Promedios con letras distintas indican diferencia significativa según la prueba de Tukey $(P \leq 0,05), n=16$.

El crecimiento del bulbo y las demás partes de la planta están directamente relacionados. Cuando la planta tiene un sistema radicular abundante se garantiza la absorción de agua y nutrientes, estos son necesarios para procesos metabólicos esenciales (Sánchez-Díaz y Aguirreolea, 2008). Así mismo, en las hojas se realiza la fotosíntesis, proceso por el cual se captan carbohidratos que formaran sólidos solubles (los cuales hacen parte del $60 \%$ al $80 \%$ de la masa seca del bulbo (Brewster, 2008; Rodríguez et al., 2009)), estos sólidos solubles totales se traslocan desde las hojas y vainas foliares hacia el bulbo en formación (Steen y Benkeblia, 2014) lo cual hace que este acumule una mayor masa.

El coeficiente que presentó un mayor desarrollo vegetal al analizar todas las partes de la planta en conjunto fue el de 1,2 de la Ev, y además presentó la mayor acumulación de masa seca del bulbo, mientras que la menor masa seca se presentó bajo coeficiente de 0,8 , el cual tuvo la menor acumulación de masa seca total.

\section{CONCLUSIONES}

El coeficiente de 1,2 de la Ev fue el tratamiento que mostró el mayor crecimiento de todos los órganos de la planta medidos. El crecimiento general de la planta de cebolla aumenta conforme aumenta la cantidad de agua desde 0,8 hasta 1,2 de la Ev, y al aplicar una cantidad mayor de agua, el crecimiento tiende a disminuir. Además, la aplicación del coeficiente de 1,2 favorece el crecimiento radicular mientras que una cantidad mayor (1,4 Ev) o menor ( 0,8 y $1,0 \mathrm{Ev})$ reduce dicho crecimiento. El coeficiente de riego de 0,8 presentó los mejores resultados durante los primeros 50 ddt (etapa inicial) en el cultivo de cebolla. Una baja aplicación de agua provoca que la senescencia empiece mas tempranamente reduciendo la acumulación de masa seca total. En cuanto al peso del bulbo, este aumenta conforme la cantidad de agua aplicada aumenta del coeficiente de 0,8 al de 1,4, aunque la cantidad de masa seca de este órgano se mantiene constante después de la aplicación de 1,2 Ev.

Conflicto de intereses: el manuscrito fue preparado y revisado con la participación de los autores, quienes declaran no tener algún conflicto de interés que coloquen en riesgo la validez de los resultados aquí presentados.

\section{REFERENCIAS BIBLIOGRÁFICAS}

Agronet. 2016. Principales departamentos productores de cebolla año 2013. En: http://agronet.gov.co/agronetweb1/Estad\%C3\%ADsticas.aspx; consulta: agosto de 2017.

Bekele, S. y K. Tilahun. 2007. Regulated deficit irrigation scheduling of onion in a semiarid region of Ethiopia. Agric. Water Manage. 89(1-2), 148-152. Doi: 10.1016/j. agwat.2007.01.002

Brewster, J.L. 2008. Onions and other vegetable Alliums. CABI International, Cambridge, MA, USA. Doi: 10.1079/9781845933999.0000 
Enciso, J., B. Wiedenfeld, J. Jifon y S. Nelson. 2009. Onion yield and quality response to two irrigation scheduling strategies. Sci. Hortic. 120(3), 301-305. Doi: 10.1016/j. scienta.2008.11.004

FAO. 2006. Evapotranspiración del cultivo: guías para la determinación de los requerimientos de agua de los cultivos. FAO, Roma, Italia.

FAO. 2016. Yield and area of dry onions in 2010. En: Statical database, http://fenix.fao.org/faostat/beta/en/?\#data/OC; consulta: agosto de 2017.

Irfan, M., S. Hayat, O. Hayat, S. Afroz y A. Ahmad. 2010. Physiological and biochemical changes in plants under waterlogging. Protoplasma 241(1), 3-17. Doi: 10.1007/ s00709-009-0098-8

Kumar, S., M. Imtiyaz y A. Kumar. 2007a. Effect of differential soil moisture and nutrient regimes on postharvest attributes of onion (Allium cepa L.). Sci. Hortic. 112(2), 121-129. Doi: 10.1016/j.scienta.2006.12.024

Kumar, S., M. Imtiyaz, A. Kumar y R. Singh. 2007b. Response of onion (Allium cepa L.) to different levels of irrigation water. Agric. Water Manag. 89(1-2), 161166. Doi: 10.1016/j.agwat.2007.01.003

Medrano, H., J. Galmés y J. Flexas. 2008. Fijación del dióxido de carbono y biosíntesis de fotoasimilados. pp. 211-126. En: Azcón-Bieto, J. y M. Talón (eds.). Fundamentos de fisiología vegetal. McGraw-Hill, Barcelona, España.

Metwally, A.K. 2011. Effect of water supply on vegetative growth and yield characteristics in onion (Allium cepa L.). Aust. J. Basic Appl. Sci. 5(12), 3016-3023.

Mishra, R., R.K. Jaiswal y D. Kumar. 2014. Management of major diseases and insect pests of onion and garlic: a comprehensive review. J. Plant Breed Crop Sci. 6(11), 160-170. Doi: 10.5897/JPBCS2014.0467

Rattin, J.E., S. Assuero, G.O. Sasso y J.A. Tognetti. 2011. Accelerated storage losses in onion subjected to water deficit during bulb filling. Sci. Hortic. 130(1), 25-31. Doi: 10.1016/j.scienta.2011.06.026
Rodríguez-Galdón, B., C. Tascón-Rodríguez, E.M. Rodríguez-Rodríguez y C. Díaz-Romero. 2009. Fructans and major compounds in onion cultivars (Allium cepa). J. Food Compos. Anal. 22(1), 25-32. Doi: 10.1016/j. jfca.2008.07.007

Sánchez-Díaz, M. y J. Aguirreolea. 2008. Transpiración y control estomático. pp. 41-56. En: Azcón-Bieto, J. y M. Talón (eds.). Fundamentos de fisiología vegetal. McGraw-Hill, Barcelona, España.

Sauter, M. 2013. Root responses to flooding. Curr. Opin. Plant Biol. 16(3), 282-286. Doi: 10.1016/j. pbi.2013.03.013

Shao, H.B., L. Chu, C.A. Jaleel y C.X. Zhao. 2008. Water-deficit stress-induced anatomical changes in higher plants. C. R. Biol. 331(3), 215-225. Doi: 10.1016/j. crvi.2008.01.002

Steen, S. y N. Benkeblia. 2014. Variation of reducing and total sugars during growth of onion tissues. Acta Hortic. 1047, 51-55. Doi: 10.17660/ActaHortic.2014.1047.3

Steffens, B. 2014. The role of ethylene and ROS in salinity, heavy metal, and flooding responses in rice. Front. Plant Sci. 5, 685. Doi: 10.3389/fpls.2014.00685

Taiz, L., E. Zeiger., I.M. Møller y A. Murphy. 2015. Plant physiology and development. $6^{\text {th }}$ ed. Sinauer Associates, Sunderland, MA, USA.

Thomas, H. 2013. Senescence, ageing and death of the whole plant. New Phytol. 197(3), 696-711. Doi: 10.1111/ nph.12047

Voesenek, L.A.C.J. y R. Sasidharan. 2013. Ethylene and oxygen signalling drive plant survival during flooding. Plant Biol. 15(3), 426-435. Doi: 10.1111/plb.12014

Zheng, J., G. Huang y J. Wang. 2012. Effects of water deficits on growth, yield and water productivity of drip-irrigated onion (Allium cepa L.) in an arid region of Northwest China. Irrig. Sci. 31(5), 995-1008. Doi: 10.1007/s00271-012-0378-5 